\title{
ANÁLISIS BIOSTÁTICO DE LAS TENSIONES ACTUANTES EN EL DENTARIO DEL OSO ANDINO (TREMARCTOS ORNATUS) DURANTE LA MORDEDURA
}

\author{
J. Fernando DEL MORAL SACHETTI 1 ,3,4, \\ Ramiro ZenTeno CárdenaS 2 \& Fátima I. LAMEda CAMACARO1,3,4 \\ ${ }^{1}$ Estación Biológica de Fauna Silvestre "Dr. Martín Vucetich”, Universidad Nacional de Jujuy, \\ ARGENTINA. \\ 2 Fundación Biodiversa Bolivia, Santa Cruz de la Sierra, BOLIVIA. ramiro.zenteno@gmail.com \\ 3Fundación AndígenA, Mérida, VENEZUELA. \\ ${ }^{4}$ Dr. Eduardo Wilde No 450, Dpto. A, V ${ }^{\text {a }}$ Soledad, C.P. 4400, Salta, ARGENTINA. \\ jfdelmoral@gmail.com
}

Del Moral S., J. F., R. Zenteno C. y F. I. Lameda C. 2009. Análisis biostático de las tensiones actuantes en el dentario del oso andino (Tremarctos ornatus) durante la mordedura. Acta Zoológica Mexicana (n. s.), 25(3): 551-567.

RESUMEN. El presente estudio tuvo la finalidad de contribuir al conocimiento biológico y biostático del oso andino (Tremarctos ornatus), el cuarto úrsido de mayor tamaño del mundo y el carnívoro más grande viviente del Neotrópico. Se obtuvieron las tensiones máximas que solicitan al dentario durante la mordedura, sus reacciones y comportamiento a los esfuerzos de corte y momentos flexores máximos, a lo largo del vano mandibular. Para ello, se analizaron 3 cráneos completos y un dentario provenientes de animales silvestres de Bolivia, utilizando 9 medidas craneanas como descriptores. Las cargas máximas de mordedura se consideraron en los caninos (c) y segundos molares superiores $\left(\mathrm{m}_{2}\right)$. Se determinaron además la intensidad de las mismas, mediante el cálculo de las tensiones más elevadas alcanzadas en dichas secciones, en función de la Tensión de Rotura $\left(\sigma_{R}=0,50 \mathrm{~kg} / \mathrm{mm}^{2}\right)$. El sistema final propuesto, responde a dos tramos de una estructura hiperestática y su resolución se efectúo mediante el Método de Aproximaciones Sucesivas de Cross. Se encontró que la fuerza máxima de mordedura en el macho es de 246,90 kg siendo 1,34 veces mayor aproximadamente, en los molares. La hembra llega a tener una mordida de $184,96 \mathrm{~kg}$ y una intensidad de molienda de $285,75 \mathrm{~kg}$. El aumento de la intensidad, en más de $80 \mathrm{~kg}$ en los molares respecto a los caninos, es directamente proporcional al gran desarrollo del músculo temporal-masetérico; como el diseño estructural óseo en la fosa homónima y la anchura del rostro a nivel molar. Estos caracteres biostáticos denotan la especialización del diseño craneodentario de la especie para soportar grandes cargas; producto de triturar y moler vegetales fibrosos duros en general, y una capacitación singular del macho para la depredación. Lo que lo posibilita al oso andino a tener una dieta variada y oportunista en su nicho ecológico, adaptable a los recursos alimenticios que el medio le ofrezca.

Palabras clave: Oso andino, Tremarctos ornatus, biostática, hiperestaticidad, método de Cross.

Recibido: 22/01/2009; aceptado: 22/09/2009. 
Del Moral S., J. F., R. Zenteno C., \& F. I. Lameda C. 2009. Biostatical analysis of the tensions acting in the jaw of the Andean bear (Tremarctos ornatus) during the bite. Acta Zoológica Mexicana ( $n$. s.), 25(3): 551-567.

ABSTRACT. This study was intended to contribute to the biological and biostatical knowledge of the Andean bear, the fourth bear largest in the world and the largest carnivore living in the Neotropics. Tensions were high, asking the tooth during, their reactions and behavior cutting efforts and maximum flexor moments, along the jaw vain. This will be analyzed 3 complete skulls and a jaw from wild animals of Bolivia, using 9 skull measurements as descriptors. The maximum bite requests were considered in the canines $(\mathrm{c})$ and second upper molars $\left(\mathrm{m}_{2}\right)$. It also determined the intensity of them, by calculating of the tensions reached higher in these sections, depending on the Fracture strength $\left(\sigma_{R}=\right.$ $0.50 \mathrm{~kg} / \mathrm{mm}^{2}$ ). The final system, responds to two portions of a hiperestatical structure and its resolution is achieved through the Moment Distribution Method by Cross. It was found that the maximum bite force in the male is $246.90 \mathrm{~kg}$ being 1.34 times higher in the molars. The female, have reached a bite of $184.96 \mathrm{~kg}$ and a milling intensity of $285.75 \mathrm{~kg}$. The increased intensity in over $80 \mathrm{~kg}$ in the molars on the canines is directly proportional to the great development of the temporalis-masseteric muscle, as the structural design of bone in the masseteric fossa and the width of face at molar. These biostatics characters reflect the design expertise craniodental of the species to withstand major stresses, product of crushing and grinding tough fibrous plants in general and training unique to the male predation. What it enables the Andean bear to have a varied diet and opportunistic in its ecological niche, adaptable to food resources that the environment offered.

Key words: Andean bear, Tremarctos ornatus, biostatic, hiperestaticity, Cross method.

\section{INTRODUCCIÓN}

El oso andino o de anteojos (Tremarctos ornatus), es el cuarto úrsido de mayor tamaño del mundo, y el carnívoro más grande viviente actualmente del Neotrópico. Su rango distributivo, se extiende desde el occidente venezolano, a lo largo de los Andes Tropicales, hasta el noroeste argentino; de 250 a 4.250 msnm (Mondolfi 1971, Peyton 1980, Yerena 1987, Nowak 1991, Weinhardt 1993, Peyton 1999, Del Moral 2007, Del Moral \& Bracho 2009).

Se ha considerado al oso andino como una especie focal, pues requiere para sobrevivir grandes superficies y es buen indicador del estado de conservación de su hábitat debido, entre otros factores por su requerimiento especializado de dieta (Mondolfi 1971, Mondolfi 1989, Peyton 1980, Suárez 1989, Rodríguez et al. 2003), la cuál contiene gran proporción de lípidos y grasas, que el animal obtiene de plantas y frutos ricos en azúcares (Peyton 1999). No obstante en contraposición, algunos estudios recientes de la mandíbula y la morfología craneodental sugieren que el oso andino está mucho menos adaptado para la herbívoria de lo que antes se suponía (Fig. 1); y se asemeja más a otros úrsidos, que al panda gigante (Ailuropoda melanoleuca) quien sí muestra tal especialización (Davis 1964, Brambell 1976, Schaller et al. 1989, Sacco \&Van Valkenburgh 2004, Christiansen 2008). En general, los úrsidos según estudios anteriores, tienen comparativamente menos diversidad craneodentaria de la que se esperaría por su gran variación de dieta (Sacco \& Van Valkenburgh 2004, Christiansen 2007, Christiansen \& Wroe 2007, Christiansen 2008). 

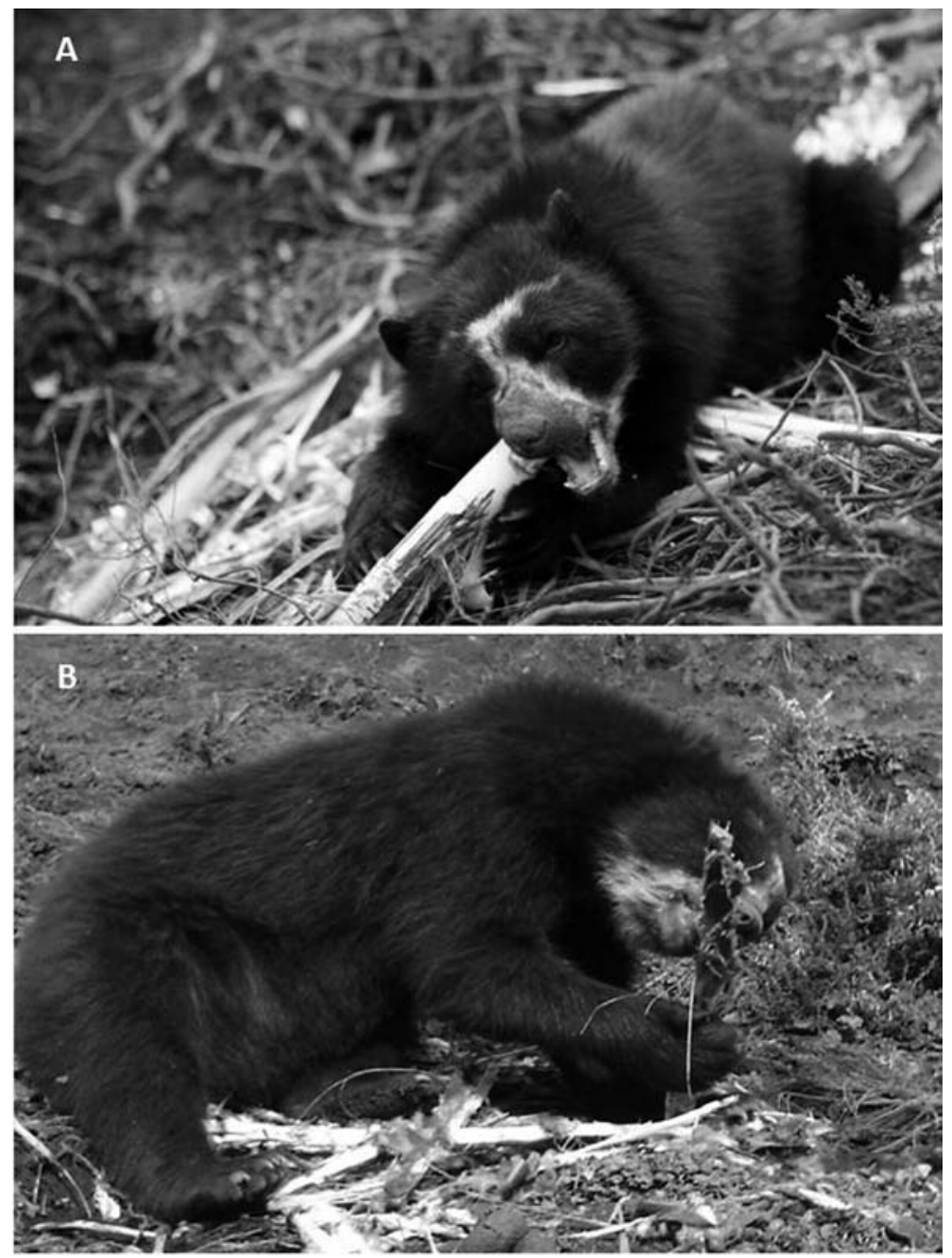

Figura 1. A) Ejemplar de oso andino (Tremarctos ornatus) durante la consumición de material fibroso vegetal, obsérvese la tendencia de molienda en movimiento lateral del dentario. B) El gran desarrollo del complejo muscular cigomático mandibular y masetérico le confieren al oso andino gran fuerza mandibular, indispensable para una dieta variada. Fotografías de Armando Castellanos.

Davis (1955) señala importantes diferencias morfológicas entre el cráneo de Tremarctos y Ursus, primordialmente en la parte posterior de la mandíbula, teniendo el cigomático ligeramente más arqueado y un hocico corto; la región frontal del cráneo, más abovedada y la mandíbula más profundas Tremarctos que Ursus. También el oso andino tiene sus músculos temporales y cigomáticos mandibulares 
comparativamente más grandes, indicando la gran fuerza mandibular en relación a su peso y morfometría que posee (Fig. 2).

En el presente estudio se determinan las fuerzas de mordedura y molienda máximas promedio para macho y hembra adultos de oso andino. Teniendo en cuenta el marcado dimorfismo sexual existente en la especie (Mondolfi 1971, Peyton 1980, Peyton 1999), se determinan las tensiones y flexiones actuantes en el dentario, durante la mordedura, y se interpretan los datos desde un punto de vista físico y biológico, tomando en cuenta su ecología alimentaria.

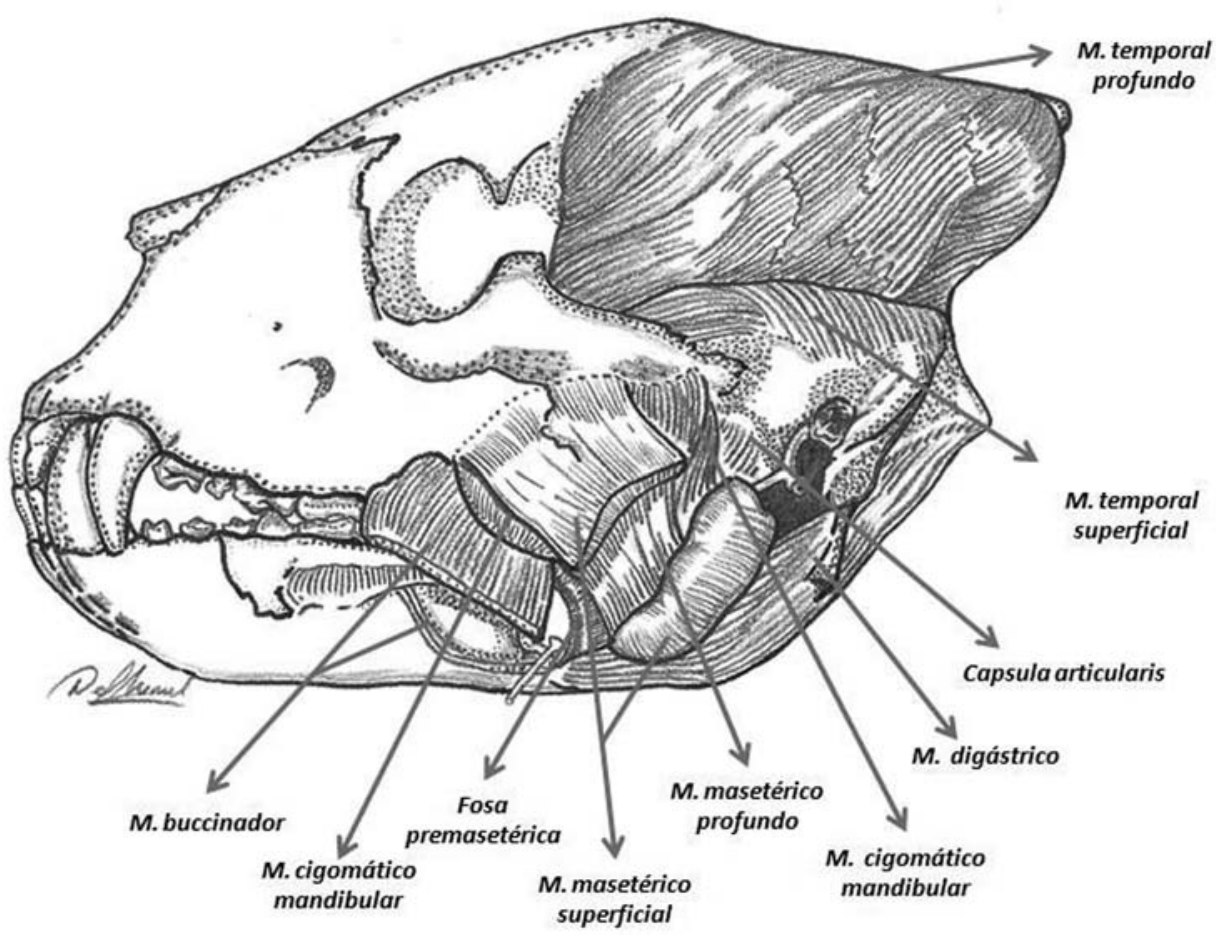

Figura 2. Musculatura masticatoria del oso andino. La mejilla se ha abierto mediante incisión continúa hacía la esquina caudal de la boca, y los buccinadores se hallan dispuestos sobre y bajo la fosa premasetérica. Véase también el importante complejo muscular: cigomático mandibularmasetérico y sus líneas de acción, en las fosas premasetérica y masetérica (Adaptado de Davis, 1955).

Dibujo de Fernando Del Moral. 


\section{MATERIALES Y MÉTODOS}

Se estudiaron tres cráneos completos y un dentario incompleto, de ejemplares adultos de oso andino procedentes de Bolivia, depositados en el Museo de Historia Natural Noel Kempff Mercado, Santa Cruz de la Sierra (MHNNKM), Bolivia. Dos ejemplares de la colección tenían ficha completa de colecta, incluyendo el sexo ( $\mathrm{N}^{\mathrm{o}}$ de Inventarios: 1244 y 1230), un cráneo completo en exhibición no poseía datos de su colecta (s/d); y el dentario $\left(\mathrm{N}^{\mathrm{o}} 3522\right)$, no tenía información acerca del sexo del ejemplar. Sin embargo, estos también se discriminaron sexualmente, de acuerdo a las luces de los descriptores empleados (existe dimorfismo sexual craneal en el oso andino, y las dimensiones son mayores en macho que en hembra), y la apertura de los arcos cigomáticos (en el macho siempre es mayor). El desgaste canino, molar y el desarrollo de las crestas parietales, se utilizaron como criterio etario. Considerándose a los ejemplares s/d y 3522, macho y hembra respectivamente. Para los análisis subsiguientes siempre se consideró a las muestras discriminadas sexualmente, a fin de obtener resultados comparativos.

Descriptores craneales. Como descriptores se seleccionaron 9 luces ó medidas craneanas (Fig. 3), tomados en base al diseño genérico de la caja craneana (afectada a las líneas de acción de los músculos masticatorios) y el dentario de la especie. Para la medición se empleó un Vernier de 0,05 mm de precisión. En la categoría luces en el dentario, se consideraron las medidas, $1_{1}$ : longitud canino-divisoria fosas premasetérica y masetérica; $1_{2}$ : longitud inicio fosa masetérica-apófisis angular; $1_{3}$ : luz inicio fosa masetérica-apófisis condilar. En la categoría alturas, en la caja craneana se consideraron las medidas, $\mathrm{p}_{1}$ : altura de caja craneana a nivel de la fosa masetérica y $\mathrm{p}_{2}$ : altura de caja craneana a nivel del apófisis condilar. Finalmente, en la categoría de áreas ó secciones palatinas en caninos (Ac) y 2do molar (Am), se consideraron las medidas, b: largo de Ac; h: altura de Ac y b": largo de Am; h": altura de Am. El promedio de los descriptores empleados en macho y hembra, se empleó luego como base del análisis (Cuadro 1).

Determinación de las fuerzas de mordedura y molienda. Se calculó la intensidad de mordedura y molienda en caninos y 2do molares superiores, respectivamente. En función de las áreas Ac $\left[\mathrm{mm}^{2}\right]$ y Am $\left[\mathrm{mm}^{2}\right]$, donde actúan estas cargas. Ambas fuerzas se consideraron puntuales en sus áreas de acción, traduciéndose en las resultantes de las tensiones de cada uno de los caninos y molares en el maxilar, correspondientemente. El planteo se consideró en equilibrio, el movimiento y densidad del tejido óseo como una constante, siguiendo premisas básicas de la mecánica estática. Al tratarse el sistema propuesto de un caso de flexión compuesta, se tomaron en cuenta para el cálculo de las fuerzas máximas [kg], que las mismas estarían dadas por las tensiones efectivas del material que deben permanecer 

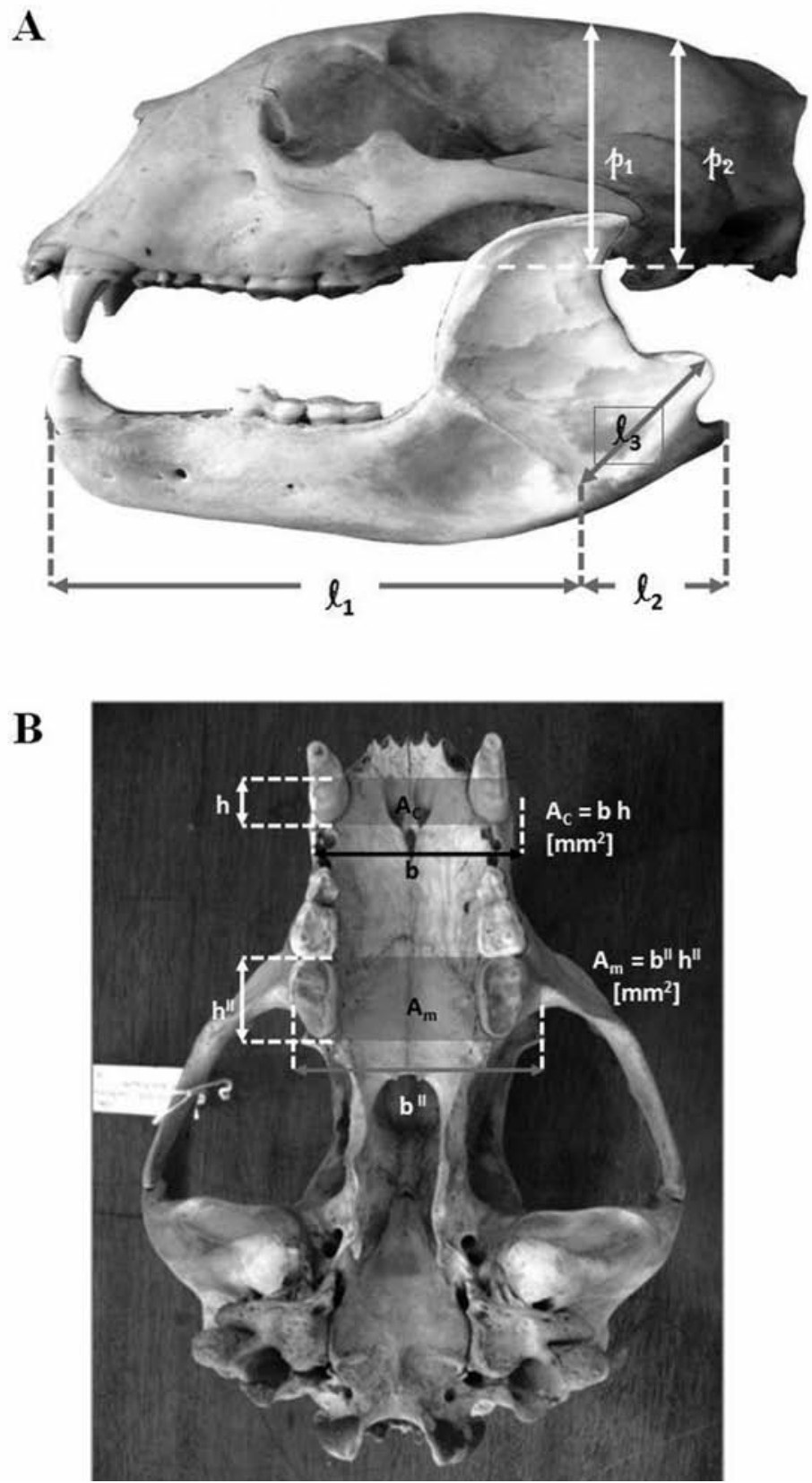

Figura 3. Vista lateral y palatina del cráneo de Tremarctos ornatus y los correspondientes descriptores empleados. A) Dimensiones de la caja craneana y del dentario. B) Dimensiones de las secciones palatinas en caninos (Ac) y molares superiores (Am). Véase definiciones en el texto. 
inferiores a la tensión admisible ó de seguridad $\left(\sigma_{\mathrm{adm}}\right)$, sin que se produzcan en este, deformaciones permanentes ó fatigas que conduzcan a su rotura:

$$
\sigma_{\mathrm{adm}=} \sigma_{\mathrm{R}} / v\left[\mathrm{~kg} / \mathrm{mm}^{2}\right]
$$

siendo: $\sigma_{\mathrm{R}}$ la tensión de rotura $\left[\mathrm{kg} / \mathrm{mm}^{2}\right]$ y $\boldsymbol{v}$ el coeficiente de seguridad, se considera de 2,2 en Ac, y 1,5 en Am. La tensión de rotura $\left(\sigma_{R}\right)$, está dada por:

$$
\sigma_{\mathrm{R}}=\mathrm{P}_{\text {máx }} / \mathrm{A}\left[\mathrm{kg} / \mathrm{mm}^{2}\right]
$$

siendo: $\mathrm{P}_{\text {máx }}$ la tensión máxima, mediante la cuál sobreviene la rotura es de $500 \mathrm{~kg}$, y A es el área de acción de $\mathrm{P}_{\text {máx }}\left[\mathrm{mm}^{2}\right]$. El cálculo de las fuerzas de mordedura $\mathrm{y}$ molienda, se obtiene mediante las expresiones:

$$
\mathrm{F}_{\text {mordedura }=} \sigma_{\mathrm{adm}} \mathrm{Ac}[\mathrm{kg}], \mathrm{F}_{\text {molienda }} \sigma_{\mathrm{adm}} \text { Am }[\mathrm{kg}] .
$$

Cuadro 1. Promedios de longitudes (mm) y descriptores empleados para 3 cráneos y 1 dentario de Tremarctos ornatus.

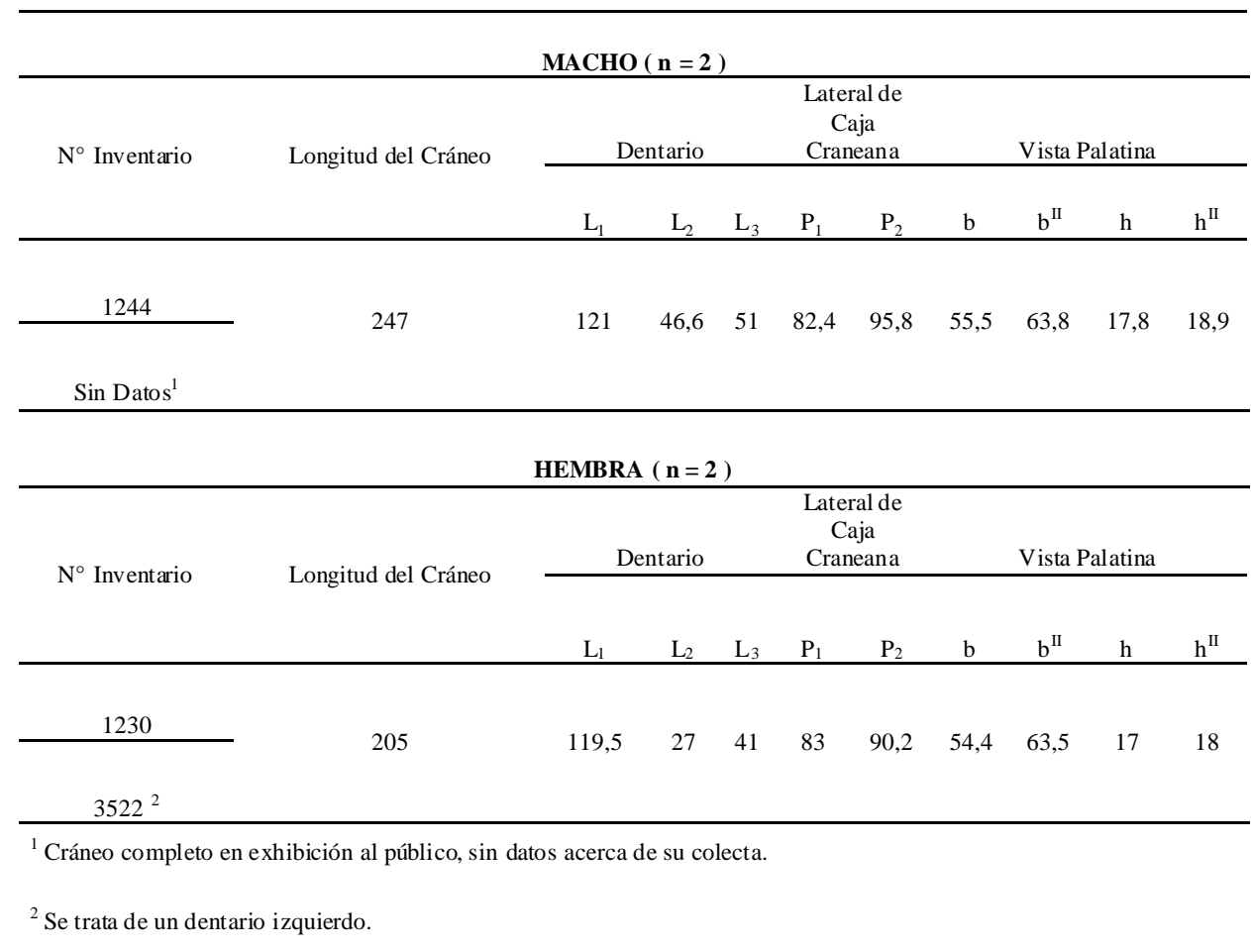


Sistema de cargas en el vano del dentario. El sistema de cargas se consideró compuesto por una carga triangular $\left(\mathrm{q}_{1}\right)$, trapezoidal $\left(\mathrm{q}_{2}\right)$ uniformemente distribuidas y dos cargas puntuales $\left(\mathrm{F}_{1} ; \mathrm{F}_{2}\right)$. La forma de la carga uniforme estuvo determinada por las líneas de acción e inserción de la musculatura masticatoria en el cráneo (Fig. 2). La intensidad de las resultantes $(\mathrm{Rq})$ de las mismas corresponde al peso propio vivo de la cabeza (con su musculatura y tegumentos), y se calculó en función del peso vivo y medidas morfométricas (longitud y altura a la cruz del cuerpo, longitud y altura de la cabeza) del taxón. Considerándose un promedio de $157,50 \mathrm{~kg}$ distribuidos en un área total de $1.232 .000 \mathrm{~mm}^{2}$ y $80 \mathrm{~kg}$ distribuidos en $840.000 \mathrm{~mm}^{2}$, entre machos y hembras, de acuerdo a pesos y dimensiones corporales reportados en la literatura (Robinson \& Redford 1986, Eisenberg \& Redford 1999, Peyton 1999). Estableciéndose relaciones sucesivas entre área de tronco y cabeza; y traduciéndose porcentualmente estos valores, se los afectó al porcentaje representativo de la cabeza en el cuerpo, obteniendo un peso propio teórico vivo de la primera. El valor de q resulta de la siguiente expresión, en cada vano:

$$
\mathrm{q}=\mathrm{Rq} / \mathrm{l}[\mathrm{kg} / \mathrm{mm}]
$$

siendo: $\mathrm{Rq}$ es la resultante de q. Peso propio de la cabeza [kg], y l: la luz en mm. Las cargas puntuales, están dadas por:

$$
\mathrm{F}=\sigma_{\mathrm{adm}} \mathrm{A} / 2[\mathrm{~kg}]
$$

El sistema final propuesto responde a dos tramos de una estructura hiperestática en la mandíbula (Fig. 4): 1) en voladizo desde el canino al apoyo empotrado A, situado a nivel de la divisoria de las fosas premasetérica y masetérica (c-A), y 2) en barra desde el apoyo articulado B, ubicado en el apófisis condilar al apoyo A (B-A). Los valores del sistema de cargas, se muestran en Cuadro 2. Por convención de signos se consideran las cargas negativas (-) y las reacciones a las mismas positivas $(+)$.

Cuadro 2. Valores de cargas puntuales $\left(\mathrm{F}_{1} ; \mathrm{F}_{2}\right)$, uniformemente distribuidas triangulares $\left(\mathrm{q}_{1}\right)$ y

\begin{tabular}{|c|c|c|c|c|}
\hline & $\begin{array}{ll}F_{1} & {[\mathrm{~kg}]}\end{array}$ & $\begin{array}{ll}F_{2} & {[\mathrm{~kg}]}\end{array}$ & $\mathrm{q}_{1} \quad[\mathrm{~kg} / \mathrm{mm}]$ & $\mathbf{q}_{2}[\mathrm{~kg} / \mathrm{mm}]$ \\
\hline МАСНО & 123,45 & 166,4 & $1,5 \times 10^{-2}$ & $1,2 \times 10^{-2}$ \\
\hline HEMBRA & 92,48 & 142,87 & $1,1 \times 10^{-2}$ & $8 \times 10^{-3}$ \\
\hline
\end{tabular}
trapezoidales $\left(\mathrm{q}_{2}\right)$ sobre el vano del dentario en macho y hembra adultos de Tremarctos ornatus. 


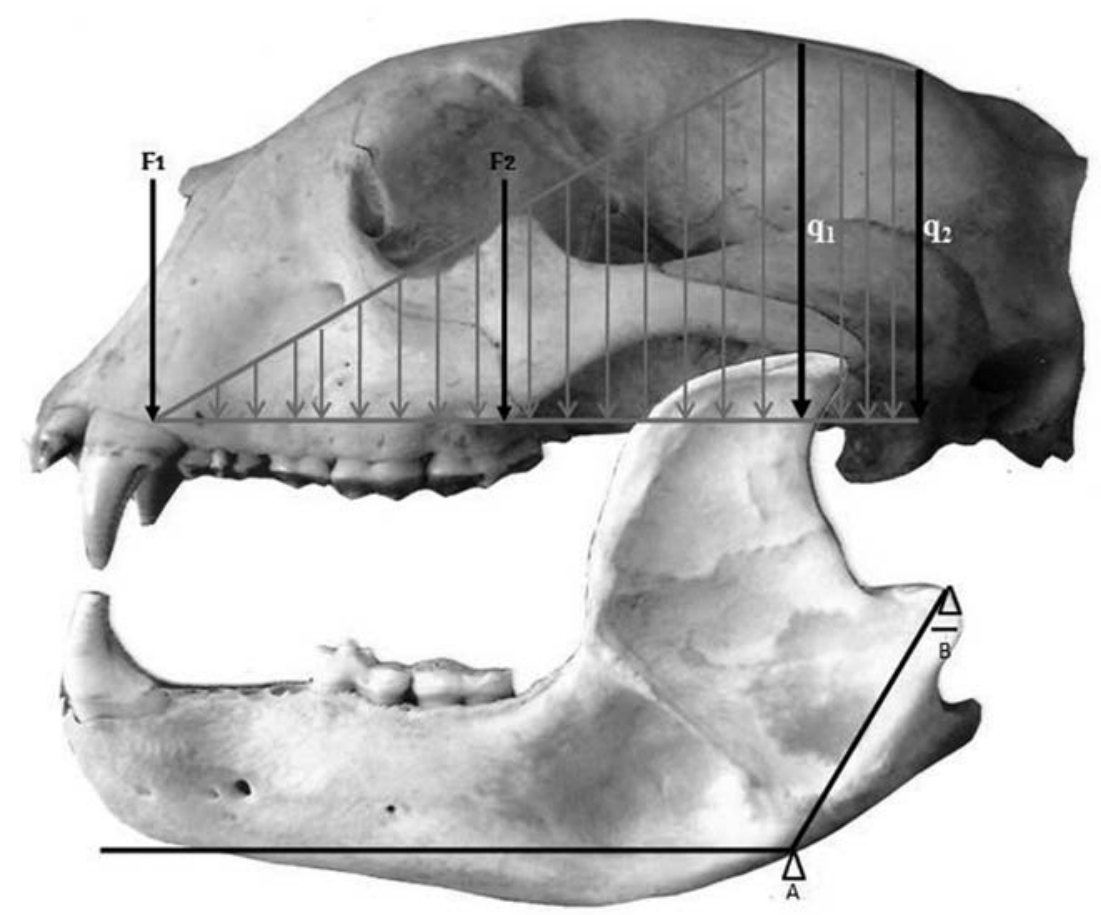

Figura 4. Esquema de cargas puntuales $\left(\mathrm{F}_{1} ; \mathrm{F}_{2}\right)$, uniformemente distribuidas triangular $\left(\mathrm{q}_{1}\right)$ y trapezoidal $\left(\mathrm{q}_{2}\right)$, sobre el vano del dentario de T. ornatus. Con apoyo considerado en A (empotrado), divisoria entre las fosas premasetérica y masetérica; y B (articulado) en el apófisis condilar. Nótese que el extremo anterior de la mandíbula al apoyo A, es funcionalmente un voladizo.

Cálculo de Momentos de Empotramiento Perfecto. El cálculo de los momentos de empotramiento perfecto $\mathrm{u}$ iniciales, en los dos tramos del vano mandibular. Estuvo dado en primer lugar, por el momento desde el voladizo c-A, que resulta de la siguiente ecuación:

$$
\text { Mto. Máx. } A_{\text {voladizo }}[\mathrm{kgmm}]=\left(-\mathrm{F}_{1} \mathrm{l}_{1}\right)+\left(-\mathrm{F}_{2} \mathrm{l}_{2}\right)+\left(-\mathrm{q}_{1} \mathrm{l}_{1}^{2 / 6}\right)
$$

El momento desde el apoyo móvil B al A, queda definido por:

siendo:

$$
\text { Mto. Máx. B-A }[\mathrm{kgmm}]=A x-\mathrm{x}^{2} / 6\left[3 \mathrm{q}_{2}+\left(\mathrm{q}_{1}-\mathrm{q}_{2}\right) \mathrm{x} / \mathrm{l}_{2}\right]
$$

$A=l_{2} / 6\left(2 q_{2}+q_{1}\right)$
$x=l_{2} / q_{1}-q_{2}-q_{2}+\left[1 / 3\left(q_{2}^{2}+q_{2} q_{1}+q_{1}^{2}\right)\right]^{1 / 2}$ 
Análisis de los datos. En el tratamiento de los datos se empleó el Método de Aproximaciones Sucesivas de Cross (Cross 1949, Timoshenko 1983, Falter 1998, Eaton 2001), mediante la obtención de parámetros básicos: coeficientes de rigideces y factores de transmisión $\left(\alpha_{\mathrm{AB}} ; \gamma_{\mathrm{A} i}\right)$ se calcularon y compararon las reacciones, momentos flexores y esfuerzos de corte en los dos tramos, del vano mandibular entre sexos. Se convino que los signos serán positivos (+) para la tracción, y negativos para la compresión (-) en los valores obtenidos. Por último, se realizaron los diagramas de esfuerzo de corte y momentos flexores, solo para el macho. Dado que por el marcado dimorfismo sexual existente, representan los valores máximos del oso andino.

\section{RESULTADOS}

Fuerzas máximas de mordedura y molienda. Se encontró que la fuerza de molienda en los segundos molares, respecto a los caninos aumentó 1,35 a 1,54 veces del macho a la hembra en relación a sus pesos y tamaños corporales, respectivamente (Fig.5). En el macho significó una tensión adicional de 85,90 kg aproximadamente, mientras que en la hembra esta tendencia fue más significativa, $100,79 \mathrm{~kg}$. Teniendo esta última 1,17 veces más tensión de molienda respecto a los caninos, que el macho. Discriminando entre caninos y molares, la relación intersexual en los primeros es más notoria que en los segundos. Estando solicitado 1,33 veces más el macho en c contra los 1,16 en $\mathrm{m}_{2}$, con respecto a la hembra.

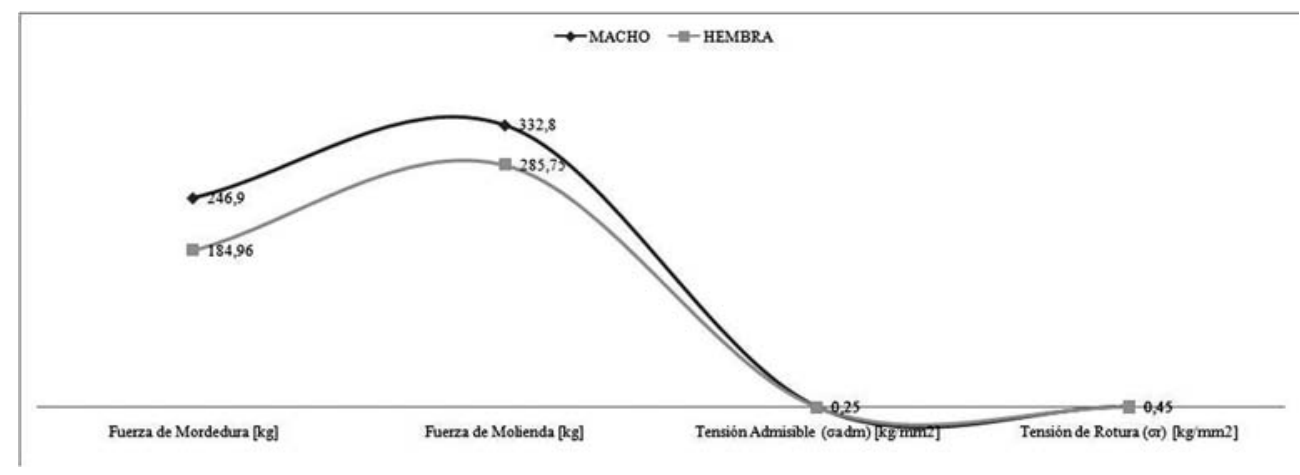

Figura 5. Distribución de fuerzas de mordedura [kg], molienda $[\mathrm{kg}]$, tensiones admisibles $\left(\sigma_{\mathrm{adm}}\right)\left[\mathrm{kg} / \mathrm{mm}^{2}\right]$ y de rotura $\left(\sigma_{\mathrm{R}}\right)\left[\mathrm{kg} / \mathrm{mm}^{2}\right]$ en el dentario de macho $\mathrm{y}$ hembra adultos de Tremarctos ornatus. 
Esfuerzos de Corte y Momentos Flexores. Las mayores tensiones de compresión se encontraron en molares desde 144,45 a $168,92 \mathrm{~kg}$, mientras que las mayores tensiones de tracción fueron las reacciones que ascendieron de 236,12 a $291,96 \mathrm{~kg}$ (de hembra a macho); entre las fosas premasetérica y masetérica (apoyo A). Rango de acción, del músculo temporal y el complejo cigomático mandibular-masetérico. En tanto en la apófisis condilar (apoyo B), descendió desde 0,28 a $0,11 \mathrm{~kg}$.

Los momentos de empotramiento máximos, fueron negativos y se encontraron en el tramo voladizo: c-A, siendo en el macho 1,21 veces mayores que en la hembra. Disminuyendo luego progresivamente desde A hasta alcanzar 0 kilogramo milímetro (kgmm), en el apoyo B. La ejecución del Diagrama de Cross nota las significativas diferencias de intensidades en reacciones, momentos y esfuerzos de corte, que solicitan el tramo c-A contra el tramo B-A (Fig. 6). Las reacciones a las cargas, denotan una ligera diferencia de $0,17 \mathrm{~kg}$ en B-A entre sexos. Las deformaciones, resultaron en: 4,62 $\mathrm{kgmm}$ en c-A y 32,20 $\mathrm{kgmm}$ en B-A mayores en la hembra, relacionada a su morfometría. En cuanto a las tensiones, estas son 60,29 kg mayores en c-A en el macho; mientras que en la hembra, son mayores en B-A con $32,37 \mathrm{~kg}$ de diferencia, sobre el primero.

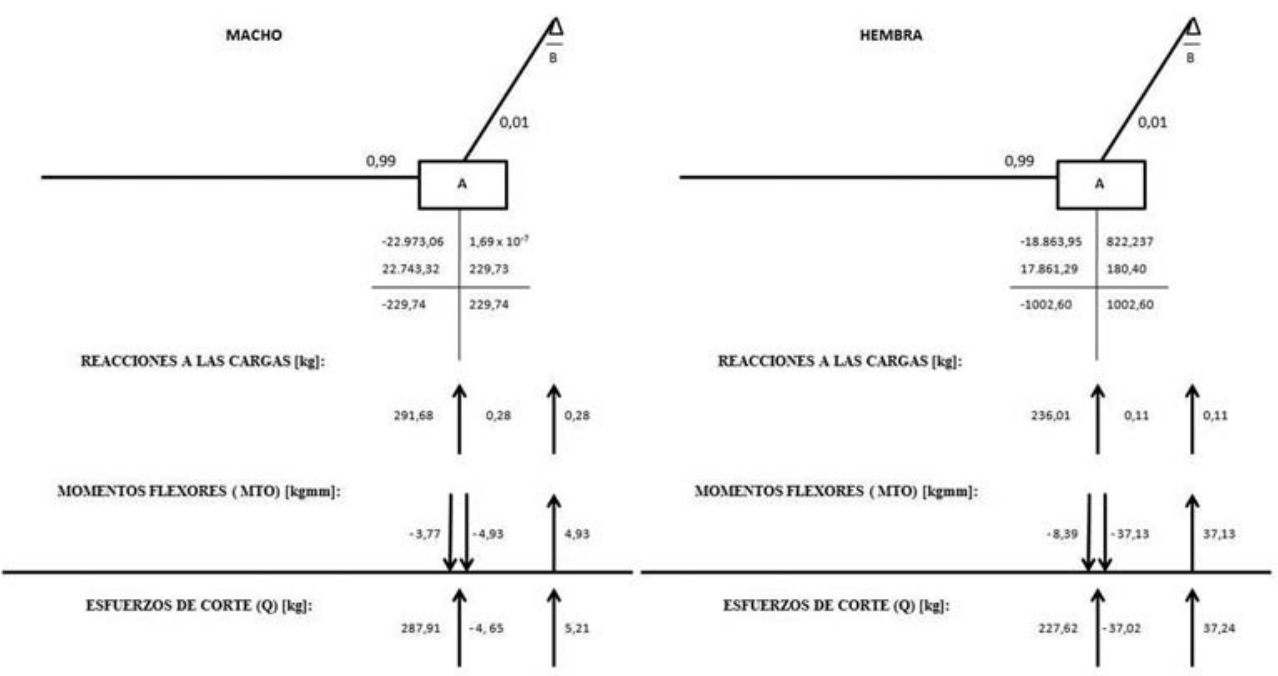

Figura 6. Diagrama de aproximaciones sucesivas de Cross y obtención de reacciones, momentos flexores y esfuerzos de corte en los dos tramos del apoyo A, en macho y hembra adultos de $T$. ornatus. 
El diagrama de esfuerzos de corte determinó que los caninos inferiores se encuentran sometidos a una compresión puntual de $123,45 \mathrm{~kg}$, disminuyendo progresivamente, afectada por la carga uniformemente distribuida $\left(\mathrm{q}_{1}\right)$ del peso propio de la cabeza en este tramo; en la porción inferior de c-A. Entre la serie molar del dentario las compresiones aumentan en $45,47 \mathrm{~kg}$, donde el músculo digástrico. En las fosas, alcanza el máximo de tracción y reacciones; en el tramo desde las fosas al apófisis condilar, los esfuerzos máximos de $\mathrm{q}_{2}$ se encontraron en los músculos cigomáticos mandibulares y temporales, cercano al punto B las tensiones son nulas y cruzan en el tramo, haciéndose ligeramente compresivas (lo cuál, es no perceptible en el diagrama de corte, comparado con las tracciones de B-A) y reaccionando con 0,28 kg en el cigomático-mandibular, vecino al apófisis (Fig. 7).

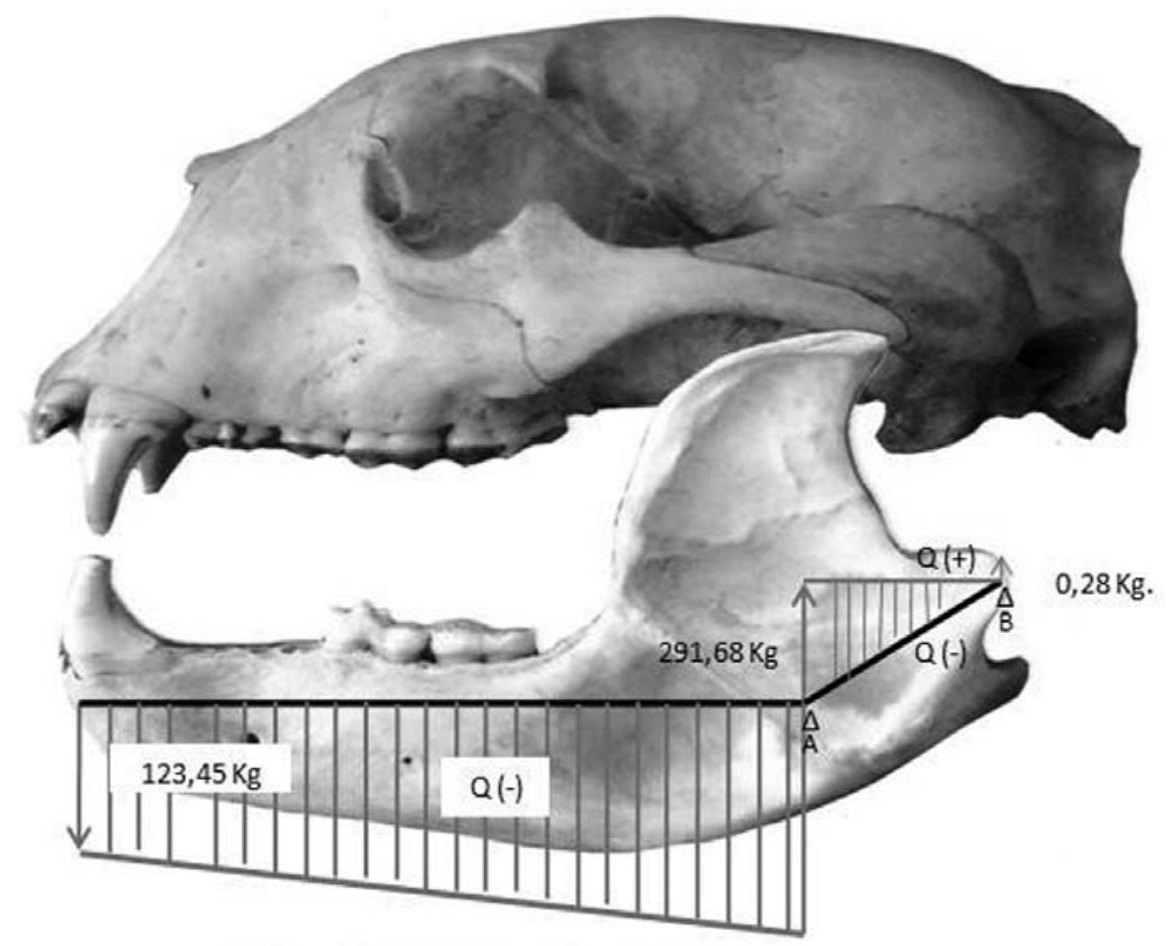

ESCALA GRAFICA DE FUERZAS [kg]

$100 \mathrm{~kg}$

Figura 7. Diagrama de esfuerzo de corte [kg] del macho adulto de T. ornatus. Nótese que el mayor esfuerzo se concentra en la divisoria de la fosa premasetérica y masetérica (apoyo A), y hacía el apófisis condilar (apoyo B) la reacción es de $0,28 \mathrm{~kg}$. 
En los momentos flexores las deformaciones son nulas en los caninos, y muestran un decremento lineal imperceptible a la flexo compresión durante su descrecimiento, con ligera tendencia parabólica en el $84 \%$ del tramo c-A. El decremento parabólico se torna evidente en el $16 \%$ restante de este tramo, alcanzando su máximo momento negativo en $\mathrm{A}$, donde el temporal y el digástrico. Hacía A-B el dentario está sometido a flexo tracción, en el rango de acción de los cigomáticos-mandibulares y el masetérico, lo que nota un sutil incremento parabólico, que alcanza su momento máximo positivo cercano al apófisis, donde el cigomático-mandibular (Fig. 8).

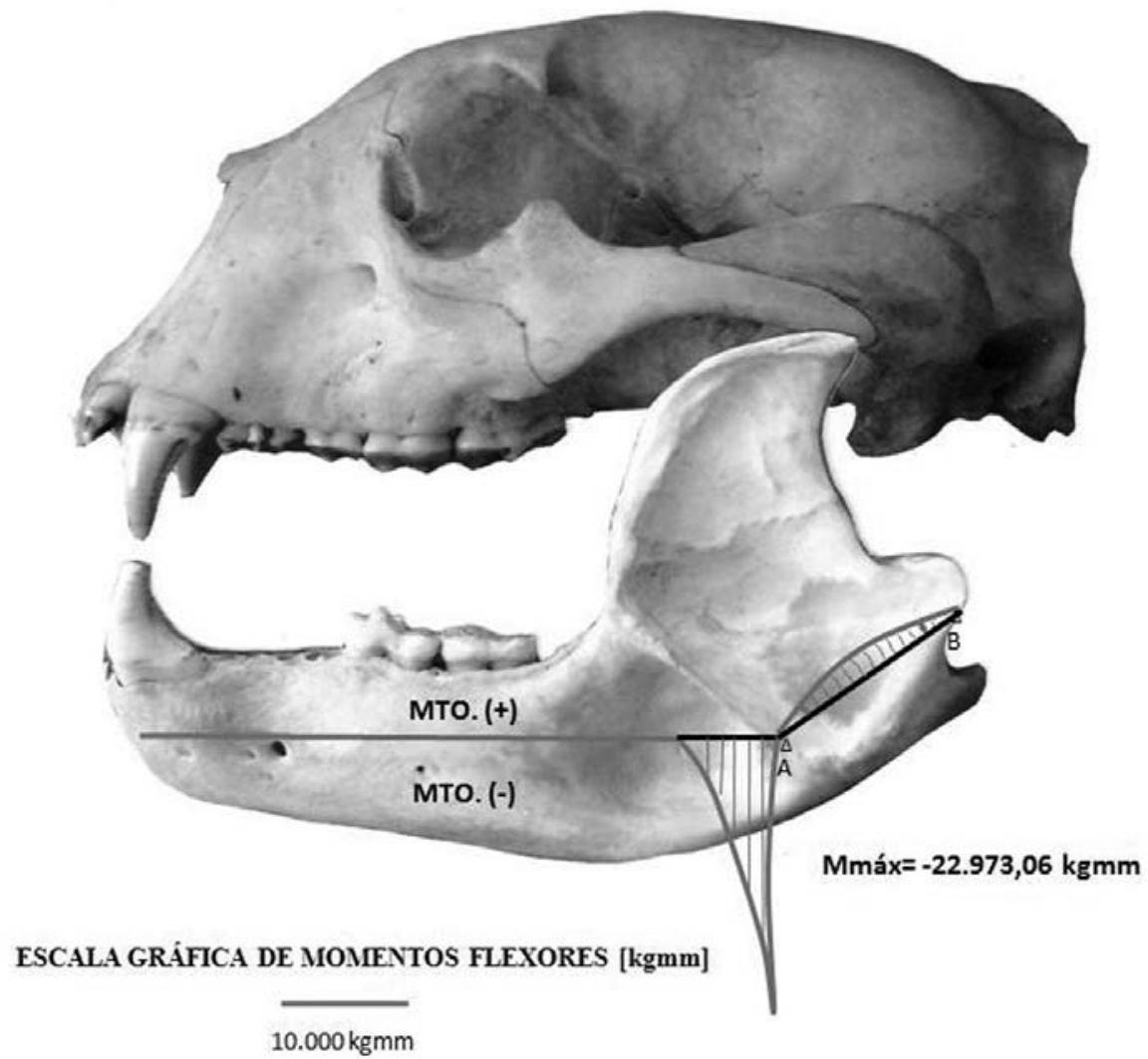

Figura 8. Diagrama de momentos flexores [kgmm] del macho adulto de T. ornatus. En los caninos el momento es de $0 \mathrm{kgmm}$ y disminuye progresivamente alcanzando su máximo negativo de flexión en la divisoria de las fosas premasetérica y masetérica. 


\section{DISCUSIÓN}

Los caninos son una característica de todos los carnívoros, que además de ser utilizados en el comportamiento agnóstico, son las armas más importantes para someter presas en la mayoría de los grupos (Van Valkenburgh \& Ruff 1987, Biknevicius \& Van Valkenburgh 1996, Van Valkenburgh 1996). Particularmente en los úrsidos, se ha señalado que parecen no seguir esta tendencia de la carnivoría; de su dependencia en caninos proporcionalmente grandes para la captura de sus presas, sinó en su morfología dental postcanina adaptada a una ecología alimentaria diversa (Biknevicius \& VanValkenburgh 1996, Christiansen 2008). En este trabajo se presenta evidencia de la elevada fuerza de mordedura del oso andino que sólo en el macho se incrementa en aproximadamente $85 \mathrm{~kg}$ hacía la serie molar. Aunque el tamaño de muestra empleado fue bajo y corresponden a un solo país de los seis en los que el taxón tiene distribución, por lo que es probable variaciones geográficas; esta tendencia muestra que la herbivoría especializada en Bromeliáceas, Bambúceas, frutos de Lauráceas y Moráceas, reportada para la especie (Mondolfi 1971, Mondolfi 1989, Peyton 1980, Suárez 1989, Peyton 1999) es relativa. Los valores encontrados entre el cociente de la fuerza de mordedura y molienda en el macho, respecto a la hembra, sugieren que este se encuentra evidentemente capacitado para eventos predatorios de presas grandes. Aunque los caninos del oso no denoten la especialización morfológica de un depredador específico como el jaguar (Panthera onca), el poder de mordedura en el macho resulta ligeramente menor al de una hembra adulta de jaguar (Del Moral, obs. per.). Esto indica también que el oso tiene que basarse más en su fuerza física si intenta someter a grandes presas, que en sus caninos (Stirling 1993, Van Valkenburgh 1993, Torres 2006, Christiansen 2008). Anecdóticamente, varios autores reportaron casos de depredación de mamíferos silvestres medianos y grandes, incluso ganado infrecuentemente; efectuados en su mayoría por machos adultos (Castellanos 2002, Galasso 2002, Goldstein et al. 2006, Torres 2006, Figueroa \& Stucchi 2009). En este mismo sentido la intensidad de molienda, de más de $300 \mathrm{~kg}$ es lo suficientemente compresiva para una eficiente masticación de elementos vegetales fibrosos, e inclusive para causar rotura en materiales óseos, de pequeñas secciones durante la alimentación.

En contraposición la hembra muestra relacionada a su morfometría, mayor fuerza de molienda. Posee un cráneo más agudo y fino, cuyo diseño en sección Ac denota menor tensión, que sin embargo se incrementa en $100 \mathrm{~kg}$ en Am, donde su sección también aumenta. Esta tendencia resulta en que la misma muestre tal vez mayor adaptación herbívora, que el macho. Además como lo fuera señalado para el panda gigante, un movimiento en bisagra y lateral de la mandíbula, representa una adaptación importante para la trituración de la comida (Sicher 1944). Dicho de otra manera, el grado de adaptación de esta a la herbivoría es 1,16 veces mayor de lo que el macho es a la carnivoría. Esto concuerda con lo señalado por Christiansen (2007, 
2008), quien encontró que las diferencias entre osos carnívoros, omnívoros y herbívoros son más evidentes en la mecánica de la mordedura, que en la forma del cráneo, la mandíbula y la morfología dental postcanina (Sacco \& Van Valkenburgh 2004).

Christiansen (2007), sugirió que el oso andino estaría menos adaptado para la herbivoría, que el panda gigante, y se encontraría más emparentado a otros úrsidos desde la morfología de la mandíbula y la mecánica craneodental (Sacco \& Van Valkenburgh 2004, Christiansen \& Wroe 2007). El presente estudio soporta esta premisa, de acuerdo a los resultados obtenidos es visible que la especie posee una resistencia a grandes tensiones y flexiones, en diferentes tramos del vano mandibular lo que le posibilita tener un amplio régimen alimenticio. Este aparente menor nivel de especialización, podría deberse en parte a su corta historia evolutiva (Christiansen 2007).

AGRADECIMIENTOS. Al personal y directivos del Museo de Historia Natural Noel Kempff Mercado (Santa Cruz de la Sierra, Bolivia) por su hospitalidad y permitirnos acceder a los ejemplares del estudio. Se agradece al biólogo Armando Castellanos (Ecuador) por su colaboración con las fotografías del oso andino, que ilustran el presente. Especialmente se retribuye al Dr. Arturo A. Canedi y la Biól. Patricia Pasini Canedi de la Estación Biológica de Fauna Silvestre "Dr. Martín Vucetich" (UNJu, Argentina), donde los primeros y terceros autores son pasantes, sin cuyo apoyo el presente trabajo no hubiera sido posible. Además se agradece a la Fundación AndígenA por el apoyo institucional brindado, a través de su presidente el Geóg. Denis Alexander Torres. Finalmente se reconoce a Jorge Moreno (Jujuy), Santiago Rocha, Ing. Roberto F. Del Moral, Prof. Angela F. Sachetti, Prof. Alcira M. Contreras (Salta, Argentina) y Rosa Adela de Camacaro (Lara, Venezuela), y se dedica a la memoria de la Prof. Rosa Adela de Camacaro (Lara, Venezuela). Dos revisores hicieron comentarios que permitieron mejorar el manuscrito.

\section{LITERATURA CITADA}

Biknevicius, A.R. \& B. Van Valkenburgh. 1996. Design for killing: Craniodental adaptations of predators. Pp. 393-428. In: J.L. Gittleman (Eds). Carnivore behaviour, ecology and evolution, Vol. 2. Cornell University Press, New York.

Brambell, M. R. 1976. The giant panda (Ailuropoda melanoleuca). Translations of the Zoological Society of London, 33:85-92.

Castellanos, A. 2002. Ataques de oso andino a ganado vacuno en la Cuenca del Río Cosanga, Ecuador. UKUKU, Boletín Informativo sobre la Conservación del Oso Andino, Año 4(1).

Christiansen, P. 2007. Evolutionary implications of bite mechanics and feeding ecology in bears. Journal of Zoology, 272:423-443.

Christiansen, P.2008. Feeding ecology and morphology of the upper canines in bears (Carnivora: Ursidae). Journal of Morphology, 269:896-908.

Christiansen, P. \& S. M. Wroe. 2007. Bite forces and evolutionary adaptations to feeding ecology in carnivores. Ecology, 88:347-358.

Cross, H. 1949. Analysis of continuous frames by distributing fixed-end movements. Pp 1-12. In: L. B. Grinter (Eds). Numerical Methods of Analysis in Engineering, Successive corrections. Macmillan Company, New York. 
Davis, D.D. 1949. The shoulder architecture of bears and other carnivores. Fieldiana Zoology, 31:285305.

. 1955. Masticatory apparatus in the spectacled bear Tremarctos ornatus. Fieldiana Zoology, $37: 25-46$.

- 1964. The giant panda: A morphological study of evolutionary mechanisms. Fieldiana Zoology, 3:1-339.

Del Moral, J.F. 2007. Proyecto Juco and the Andean Bear. Pp. 151. In: Proceedings of 18th International Conference on Bear Research and Management. International Association for Bear Research and Management (IBA). Monterrey, Nuevo León.

Del Moral, J. F. \& A. E. Bracho. 2009. Indicios indirectos de la presencia del oso andino (Tremarctos ornatus Cuvier, 1825) en el noroeste de Argentina. Revista del Museo Argentino de Ciencias Naturales, 11:69-76.

Eaton, L. K. 2001. Hardy Cross and the moment distribution method. Nexus Network Journal, Vol.3, $\mathrm{N}^{\mathrm{o}}$ 2. http://www.nexusjournal.com/Eaton.html

Eisenberg, J. F. \& K. H. Redford. 1999. Mammals of the Neotropics, the central Neotropics: Ecuador, Peru, Bolivia, Brazil, Vol.3. University of Chicago Press, Chicago.

Falter, H. 1998. The influence of mathematics on the development of structural form. Pp. 51-64. In: Kim Williams (Eds). Nexus II: Architecture and Mathematics. Edizioni dell'Erba Fucecchio, Florence.

Figueroa, J. \& M. Stucchi. 2009. El Oso Andino: alcances sobre su historia natural. Asociación para la Investigación y Conservación de la Biodiversidad-AICB, Lima.

Galasso, L. 2002. The spectacled bear's impact on livestock and crops and use of remnant forest fruit trees in a human altered landscape in Ecuador. Thesis, University of Wisconsin, Wisconsin.

Goldstein I., S. Paisley, R. Wallace, J. P. Jorgenson, F. Cuesta \& A. Castellanos. 2006. Andean bearlivestock conflicts: A review. Ursus, 17:8-15.

Mondolfi, E. 1971. El oso frontino (Tremarctos ornatus). Defensa de la Naturaleza, 1:31-35.

Mondolfi, E.1989. Notes on the distribution, habits, food habits, status and conservation of the spectacled bear in Venezuela. Mammalia, 53:525-544.

Nowak, R.M. 1991. Walker's mammals of the world, Vol. 1 and 2. Johns Hopkins University Press, Baltimore.

Peyton, B. 1980. Ecology, distribution and food habits of spectacled bears (Tremarctos ornatus) in Peru. Journal of Mammalogy, 61:639-652.

- 1999. Spectacled Bear Conservation Action Plan. Pp.157-198. In: C. Servheen, S. Herrero and B. Peyton (Eds). Bears-status survey and conservation action plan. IUCN/SSC, Bear and Polar Bear Specialist Groups IUCN, Gland, Switzerland and Cambridge.

Robinson, J. G. \& K. H. Redford. 1986. Body size, diet and population density of Neotropical forest mammals. The American Naturalist, 128: 665-680.

Rodríguez, D., F. Cuesta, I. Goldstein, A. Bracho, L. Naranjo \& O. Hernández. 2003. Estrategia Ecorregional para la conservación del oso andino (Tremarctos ornatus) en los Andes del Norte. WWF Colombia, Fundación Wii, Ecociencia and Wildlife Conservation Society (Eds). Colombia.

Sacco, T. \& B. Van Valkenburgh. 2004. Ecomorphological indicators of feeding behaviour in the bears (Carnivora: Ursidae). Journal of Zoology, 263:41-54.

Schaller, G. B., T. Quitao, K.G. Johnson, X. Wang, H. Shen \& J. Hu. 1989. The feeding ecology of giant pandas and Asiatic black bears in the Tangjiahe Reserve, China. Pp. 212-241. In: J. L. Gittleman (Eds). Carnivore behaviour, ecology and evolution, Vol.1. Cornell University Press, New York. 
Sicher, H. 1944. Masticatory apparatus in the giant panda and the bears. Field Museum of Natural History (Zoological Series), 29:61-73.

Stirling, I. 1993. The living bears. Pp. 36-49. In: I. Stirling (Eds). Bears: Majestic creatures of the wild. Harper Collins Publishers, London.

Suárez, L. 1989. Seasonal distribution and food habits of the spectacled bear (Tremarctos ornatus) in the highlands of Ecuador. Studies on Neotropical Fauna and Environment, 23:133-136.

Timoshenko, S. 1983. History of the strength of materials, with a brief account of the history of theory of elasticity and theory of structures. Mc Graw-Hill, New York.

Torres, D. 2006. Guía Básica para la identificación de señales de presencia de oso frontino (Tremarctos ornatus) en los Andes Venezolanos. Fundación Andígena, Mérida.

Van Valkenburgh, B. \& C. B. Ruff. 1987. Canine tooth strenght and killing behaviour in large carnivores. Journal of Zoology, 212:379-397.

Van Valkenburgh, B. 1993. The biology of bears. Pp. 50-61. In: I. Stirling (Eds). Bears: Majestic creatures of the wild. Harper Collins Publishers, London.

Van Valkenburgh, B. 1996. Feeding behaviour in free-ranging, large African carnivores. Journal of Mammalogy, 77: 240-254.

Yerena, E. 1987. Distribución pasada y contemporánea de los úrsidos en América del Sur. Seminario EA-7154, Dpto. de Estudios Ambientales, Universidad Simón Bolívar, Caracas.

Weinhardt, D. 1993. The spectacled bear. Pp. 134-139. In: I. Stirling (Eds). Bears: Majestic creatures of the wild. Harper Collins Publishers, London. 